\title{
Non-grey dimming events of KIC 8462852 from GTC spectrophotometry ${ }^{\star}$
}

\author{
Hans J. Deeg ${ }^{1,2}$, Roi Alonso ${ }^{1,2}$, David Nespral ${ }^{1,2}$, and Tabetha S. Boyajian ${ }^{3}$ \\ ${ }^{1}$ Instituto de Astrofísica de Canarias, C. Via Lactea S/N, 38205 La Laguna, Tenerife, Spain \\ e-mail: hdeeg@iac.es \\ ${ }^{2}$ Universidad de La Laguna, Dept. de Astrofísica, 38206 La Laguna, Tenerife, Spain \\ ${ }^{3}$ Louisiana State University, Dept of Physics \& Astronomy, Baton Rouge, LA 70803, USA \\ Received 12 December 2017 / Accepted 1 January 2018
}

\begin{abstract}
We report ground-based spectrophotometry of KIC 8462852 obtained during its first dimming events since the end of the Kepler mission. The dimmings show a clear colour signature and are deeper in visual blue than in red wavelengths. The wavelength dependency of the flux loss can be described with an absorption Ångström coefficient of $2.19 \pm 0.45$, which is compatible with absorption by optically thin dust with particle sizes of the order of 0.0015 to $0.15 \mu \mathrm{m}$. These particles would be smaller than is required to be resistant against blow-out by radiation pressure when close to the star. During occultation events, these particles must be replenished from a comoving body on time-scales of days. If dust is indeed the source of the dimming events of KIC 8462852, deeper dimming events should show more neutral colours, as is expected from optically thick absorbers.
\end{abstract}

Key words. stars: individual: KIC 8462852 - stars: peculiar - opacity - interplanetary medium

\section{Introduction}

The detection of the strange brightness variations of the star KIC 8462852, or Boyajian's star (from here on denoted simply as "KIC8462"), in post-mission data from the Kepler satellite reported by Boyajian et al. (2016) has given rise to one of the most enigmatic astronomic discoveries in recent time. During the coverage by Kepler in the years 2009-2013, this star displayed two periods of strong dips in brightness, both lasting a few weeks and separated by about two years. It is a normal F star in most respects and quiescent most of the time, but has also been claimed to have undergone a $20 \%$ brightness loss between 1890 and the present (Schaefer 2016), as well as a $0.34 \%$ brightness loss per year during the four years of the Kepler mission, with some periods of stronger decrease (Montet \& Simon 2016). Several families of explanations for its dimming events have been discussed by Wright \& Sigurdsson (2016), although none of them was entirely satisfying. While most attention has been given to occultations from a swarm of very large comet-like objects (Boyajian et al. 2016; Bodman \& Quillen 2016; Wyatt et al. 2018), further recent efforts have focused on explaining the brightness variations in KIC8462 through intervening dust that arises from dust-enshrouded planetesimals (Neslušan \& Budaj 2017) or through circumstellar dust (Meng et al. 2017).

Since the strange behaviour of KIC8462 was not discovered until long after the end of the Kepler mission, the only data that have been available from its dimming events was the single-band photometry from that mission. Arguing that

\footnotetext{
* The individual reduced spectra of the target and reference stars are only available at the CDS via anonymous ftp to cdsarc.u-strasbg.fr $(130.79 .128 .5)$ or via http://cdsarc.u-strasbg.fr/viz-bin/qcat?J/A+A/610/L12
}

colour signatures during the dimming events of KIC8462 might be indicative of their nature, we obtained target-of-opportunity observing time with the Gran Telescopio Canarias (GTC), in order to perform multicolour spectrophotometry of KIC8462, whenever this target would exhibit relevant new dimming events. Fortunately, KIC8462 exhibited several such events between May and December 2017, with flux variations of up to $2 \%$, which we observed at 16 different occasions. Here we present these observations and their analysis with regard to the hypothesis of dusty occulters. These observations were also part of a larger community-wide follow-up program whose results are published by Boyajian et al. (2018, B18).

\section{Observations}

Spectrophotometric observations of the target were obtained with the GTC's OSIRIS long-slit spectrograph using the R1000R grism, a slit width of $12^{\prime \prime}$, and a $2 \times 2$ binning of the CCD. These data were obtained through the target-of-opportunity programs 89-GTC54/17A and 71-GTC57/17B, which were triggered for the first time on May 17, 2017, after receiving an alert of a possible variation in the spectrum of KIC8462 through the community monitoring program described in B18. This alert enabled us to commence with observations with the GTC at the onset of the first known dimming event since the end of the Kepler mission. Until December 5, 2017, we acquired 16 pointings on KIC8462, covering several further dimming events, as well as moments when the target had returned almost to the brightness level prior to these events (see Table 1 for dates). Unfortunately, a technical defect forced a shut-down of the GTC for several weeks between mid-September and late November 2017, during which KIC8462 exhibited fluxes that were up to $1 \%$ higher 
Table 1. Observed fluxes at each pointing in five wavelengths bands, indicated by their central wavelengths in $\mathrm{nm}$.

\begin{tabular}{clccccc}
\hline \hline $\mathrm{Nr}$ & $\mathrm{BJD}-2400000$ & $F_{556}$ & $F_{618}$ & $F_{709}$ & $F_{792}$ & $F_{868}$ \\
\hline $1^{a}$ & 57890.6940 & $0.9942 \pm 0.0020$ & $0.9984 \pm 0.0010$ & $1.0046 \pm 0.0011$ & $1.0010 \pm 0.0010$ & $0.9986 \pm 0.0012$ \\
2 & 57891.6709 & $0.9917 \pm 0.0018$ & $0.9946 \pm 0.0018$ & $0.9976 \pm 0.0028$ & $0.9971 \pm 0.0027$ & $0.9961 \pm 0.0013$ \\
3 & 57893.6921 & $0.9879 \pm 0.0008$ & $0.9901 \pm 0.0008$ & $0.9936 \pm 0.0010$ & $0.9948 \pm 0.0010$ & $0.9943 \pm 0.0009$ \\
4 & 57895.6698 & $0.9933 \pm 0.0010$ & $0.9951 \pm 0.0008$ & $0.9961 \pm 0.0011$ & $0.9967 \pm 0.0007$ & $0.9960 \pm 0.0007$ \\
5 & 57896.6644 & $0.9950 \pm 0.0013$ & $0.9963 \pm 0.0009$ & $0.9954 \pm 0.0012$ & $0.9954 \pm 0.0009$ & $0.9971 \pm 0.0009$ \\
6 & 57897.6662 & $0.9976 \pm 0.0013$ & $1.0002 \pm 0.0010$ & $1.0002 \pm 0.0015$ & $1.0011 \pm 0.0011$ & $1.0013 \pm 0.0013$ \\
7 & 57902.7096 & $0.9969 \pm 0.0015$ & $0.9994 \pm 0.0010$ & $1.0004 \pm 0.0018$ & $0.9994 \pm 0.0017$ & $1.0005 \pm 0.0014$ \\
8 & 57921.6949 & $0.9813 \pm 0.0009$ & $0.9861 \pm 0.0007$ & $0.9912 \pm 0.0012$ & $0.9940 \pm 0.0011$ & $0.9933 \pm 0.0013$ \\
9 & 57922.5738 & $0.9898 \pm 0.0013$ & $0.9926 \pm 0.0009$ & $0.9941 \pm 0.0015$ & $0.9957 \pm 0.0013$ & $0.9956 \pm 0.0009$ \\
10 & 57980.5654 & $0.9938 \pm 0.0010$ & $0.9945 \pm 0.0015$ & $0.9945 \pm 0.0021$ & $0.9961 \pm 0.0021$ & $0.9970 \pm 0.0015$ \\
11 & 57985.6109 & $0.9995 \pm 0.0025$ & $1.0008 \pm 0.0020$ & $1.0038 \pm 0.0018$ & $1.0026 \pm 0.0012$ & $1.0007 \pm 0.0019$ \\
$12^{b}$ & 57986.6427 & $0.9991 \pm 0.0015$ & $1.0043 \pm 0.0014$ & $1.0129 \pm 0.0019$ & $1.0063 \pm 0.0009$ & $1.0017 \pm 0.0012$ \\
13 & 58007.3854 & $0.9780 \pm 0.0013$ & $0.9811 \pm 0.0008$ & $0.9855 \pm 0.0013$ & $0.9882 \pm 0.0010$ & $0.9879 \pm 0.0014$ \\
14 & 58091.3543 & $1.0051 \pm 0.0010$ & $1.0026 \pm 0.0015$ & $1.0021 \pm 0.0021$ & $1.0010 \pm 0.0027$ & $0.9998 \pm 0.0014$ \\
15 & 58092.3331 & $1.0044 \pm 0.0015$ & $1.0007 \pm 0.0016$ & $0.9996 \pm 0.0010$ & $0.9994 \pm 0.0022$ & $0.9988 \pm 0.0014$ \\
16 & 58093.3502 & $1.0011 \pm 0.0013$ & $0.9997 \pm 0.0013$ & $0.9997 \pm 0.0015$ & $1.0001 \pm 0.0019$ & $0.9994 \pm 0.0018$ \\
\hline
\end{tabular}

Notes. The BJD times refer to the central time of each pointing. ${ }^{(a)}$ Data not used in analysis due to large scatter from varying exposure times.

(b) Data not used due to poor sky transparency.

than previously recorded (see Fig. 1). The three GTC pointings in early December were taken when the target had nearly returned to the assumed quiescent flux. Each pointing consisted of a time series of about 30 min duration, based on a series of exposures with an open shutter time of $20 \mathrm{~s}$ and cycle-time of about $45 \mathrm{~s}$ (varying exposure times were used in the first pointing). A nearby star of similar brightness but redder colours (KIC $8462763, \mathrm{~V}=11.86$, with $T_{\text {eff }}=4730 \mathrm{~K}$, from the Kepler Input Catalogue (Brown et al. 2011), vs. the $T_{\text {eff }}=6750 \mathrm{~K}$ of KIC8462, from Boyajian et al. 2016) was included in the same entrance slit and was used as reference in the photometric analysis.

\section{Extraction of multicolour photometry}

The reduction procedure followed the precepts of Alonso et al. (2016), with the difference that a zero-order spectrum was not recorded while using the R1000R grism. Instead, the shifts of the spectra in the wavelength region were estimated and corrected a posteriori using the $\mathrm{H}_{\alpha}$ line for both the target and comparison. An aperture of 44 pixels width was used to extract the flux from the spectra, and the sky background was estimated in regions close to the spectra. This relatively wide aperture was needed as the spectra were slightly defocused, both to avoid saturation of the reference star and to try to minimize the errors due to inefficient flat-field correction.

The extracted spectra were divided into five wavelength bands that avoid significant telluric and stellar lines. The selected regions are presented in Fig. 2. Final fluxes in each band were obtained from the average flux during the time series acquired at each of the pointings. The corresponding flux errors are the uncertainties of the mean of the time series, that is, their standard deviation divided by $\sqrt{N}$, were $N$ is the number of individual measures in a given time series. The fluxes and their errors were then normalised to the average flux from pointings $6,7,15$, and 16, when KIC8462 had a brightness close to the one before the first dimming event. This normalisation procedure was performed independently for each of the bands. Table 1 indicates

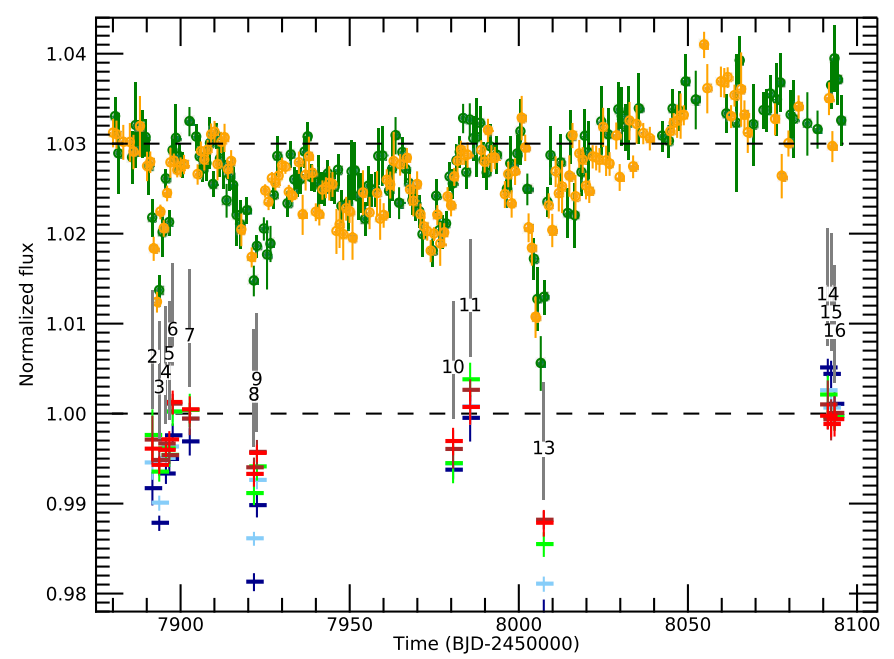

Fig. 1. Normalised fluxes of KIC 8462852 from May 6 to December 8, 2017, from the LCO and GTC. The upper light curve shows nightly photometry in the $r^{\prime}$ filter from the $0.4 \mathrm{~m}$ LCO telescopes in Tenerife (green) and Hawaii (orange), shifted upwards by 0.03 flux units for clearer display. The assumed quiescent flux is indicated by the dashed line. The lower symbols show the corresponding GTC narrow-band fluxes with their errors. The crosses' colours correspond to the wavelength ranges indicated by Fig. 2. The vertical lines indicate the time of each numbered pointing. We only show the 14 pointings used in the analysis.

these averaged and normalised fluxes, while Fig. 1 displays them together with nightly photometry obtained by two $0.4 \mathrm{~m}$ telescopes of the Las Cumbres Observatory (LCO) network. Two GTC pointings, 1 and 12, were rejected in the further analysis since their colours presented a much larger scatter than the others, with either of these pointings increasing the $\chi^{2}$ of the fitting reported in Sect. 4 by more than $40 \%$. This was due to inconsistent exposure times during the first pointing, and poor sky conditions at the twelfth pointing. As is evident, the bluer 


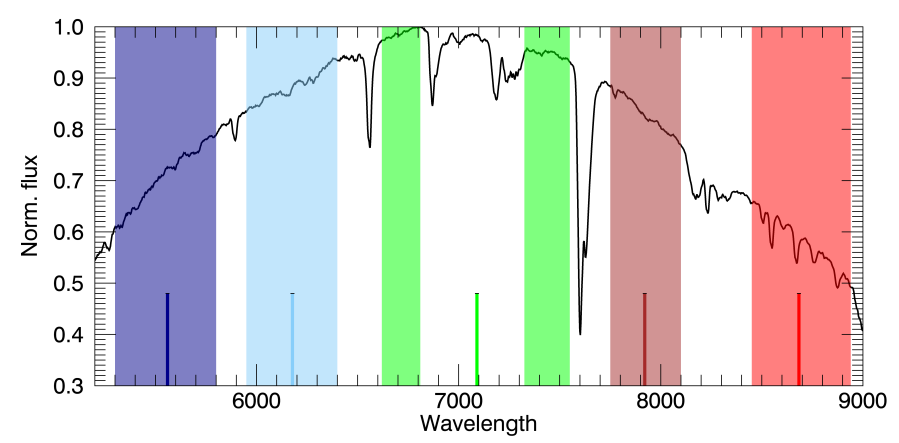

Fig. 2. Spectrum of KIC 8462852 with the featureless ranges that were extracted for photometry. The flux-weighted central wavelengths of the bands are $556 \mathrm{~nm}$ (blue), $618 \mathrm{~nm}$ (light blue), $709 \mathrm{~nm}$ (lime), $792 \mathrm{~nm}$ (brown), and $868 \mathrm{~nm}$ (red); they are indicated by the vertical lines at the bottom of the plot.

wavelength bands from the GTC data undergo deeper dimming events (see also Fig. 3); this is analysed in the next section. The LCO data are described in the parallel publication about the community follow-up effort (B18).

\section{Assessment of the spectrophotometry}

The analysis of the wavelength-dependent photometric data also builds on the method of Alonso et al. (2016), who analysed a similar data set from GTC for the presence of colour signatures. In brief, an optically thin occulter of uniform optical depth is assumed to be responsible for the dimming events. This occulter covers a fraction $f$ of the surface of the star. The fractional depth of a dimming event is then given by $D_{n}=\tau_{n} f$, where $D_{n}$ and $\tau_{n}$ refer to the depth and optical depth, respectively, at the wavelength $\lambda_{n}$. The occulter is assumed to consist of dustlike particles with an extinction cross-section $\sigma_{\text {ext }}$ for which a wavelength dependency of the form $\sigma_{\text {ext }} \propto \tau \propto \lambda^{\alpha}$ is assumed, leading to

$D_{n} \propto\left(\lambda_{n}\right)^{-\alpha}$

For a dust column of fixed density and composition, this defines the exponent $\alpha$, also known as the absorption Angström coefficient (AAC; Moosmüller 2011). The use of the AAC is preferable over the quoting of extinction or opacity ratios between different wavelengths, since Ångström coefficients (Ångström 1929) do not have a first-order dependency on the wavelength ratio, as do these ratios.

Alonso et al. (2016) derived their Ångström coefficient of similar spectrophotometry from the slope of measures of $D$ at two different wavelengths (see their Eqs. (3)-(5)). When we repeated this procedure with the data of KIC8462, the $\alpha$ values varied strongly in dependence on the wavelengths being paired, however, and an $\alpha$ value common to our five bands was very sensitive to each pairing's weight. We therefore developed a procedure that derives a common value of $\alpha$ from a simultaneous fit to the acquired depths at all bands, as follows.

Using the relation between depth and wavelength of Eq. (1), we assume that the modelled depths $D_{\bmod , n, i}$ at the wavelengths $\lambda_{n}$ relate to reference depths $D_{w, i}$ at the wavelength $\lambda_{w}$ with

$\frac{D_{\mathrm{mod}, n, i}+\Delta}{D_{w, i}+\Delta}=\left(\frac{\lambda_{n}}{\lambda_{w}}\right)^{-\alpha}$,

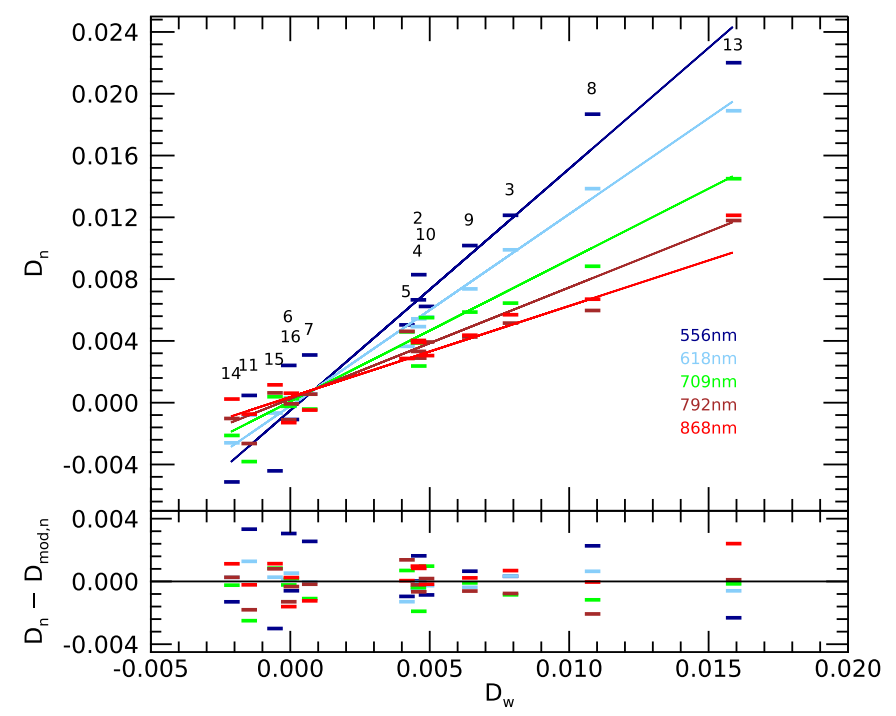

Fig. 3. Measured depths $D_{n}$ in the five colour bands, indicated by the same colours as in Fig. 2, vs. the white (average) depths $D_{w}$ for the 14 pointings that were analysed. The straight lines indicate the joint fit to all bands, with the best common value for the AAC of $\alpha=2.19$. The lower panel shows the residuals on the same scale. Error bars are omitted for clarity.

where the indices $n$ indicate the bands and $i$ stands for each telescope pointing. $\Delta$ is an offset common to all wavelengths, which accounts for any uncertainties in the global flux normalisation. The reference values $D_{w, i}$ were obtained for each pointing $i$ from the average of the depths of all the five bands. For the model fitting function, Eq. (2) was resolved for $D_{\bmod , n, i}$ and a $\chi^{2}$ minimisation was performed for

$\chi^{2}=\sum \frac{\left(D_{\bmod , n, i}(\alpha, \Delta)-D_{n, i}\right)^{2}}{\sigma_{D_{n, i}}^{2}}$,

with three free parameters to be fitted, namely $\alpha, \Delta$, and $\lambda_{w}$. The initial value for the reference wavelength $\lambda_{w}$ was the fluxweighted mean from all five band-passes, at $709 \mathrm{~nm}$. Including $\lambda_{w}$ as a free parameter led to a marked improvement in the fit, as shifts in $\lambda_{w}$ correspond to the weighting of the individual bands' fluxes when obtaining $D_{w}$. From an AMOEBA minimisation of the above equation, a best-fitting value for the AAC was obtained as

$\alpha=2.19 \pm 0.45$

with an offset of $\Delta=-0.0009 \pm 00005$ in normalised flux units, and a wavelength $\lambda_{w}=682 \pm 15 \mu \mathrm{m}$. The relation between the depths $D_{n, i}$ in the various wavelengths vs. the reference depths $D_{w, i}$ is shown in Fig. 3, together with the best-fitting model values $D_{\bmod , n}\left(D_{w}\right)$ for each wavelength $\lambda_{n}$. In this figure, $\Delta$ is visible as the amount by which the node among the model lines is shifted against the zero-points of the $D_{n, i}$ and $D_{w, i}$, whereas changes in the reference wavelength $\lambda_{w}$ correspond to changes in the gradients of the entire set of model lines.

We noted above that the quoted errors of the $D_{n, i}$ are the error of the mean of the time series that led to each flux value. In addition to these errors, we expect difficult-to-quantify errors from the flux calibration of the target against the reference star, and errors from other sources, such as flat-fielding errors or effects from differential atmospheric transmission. The model with the best-fitting values of $\alpha, \Delta$, and $\lambda_{w}$ had a reduced chi-square of 


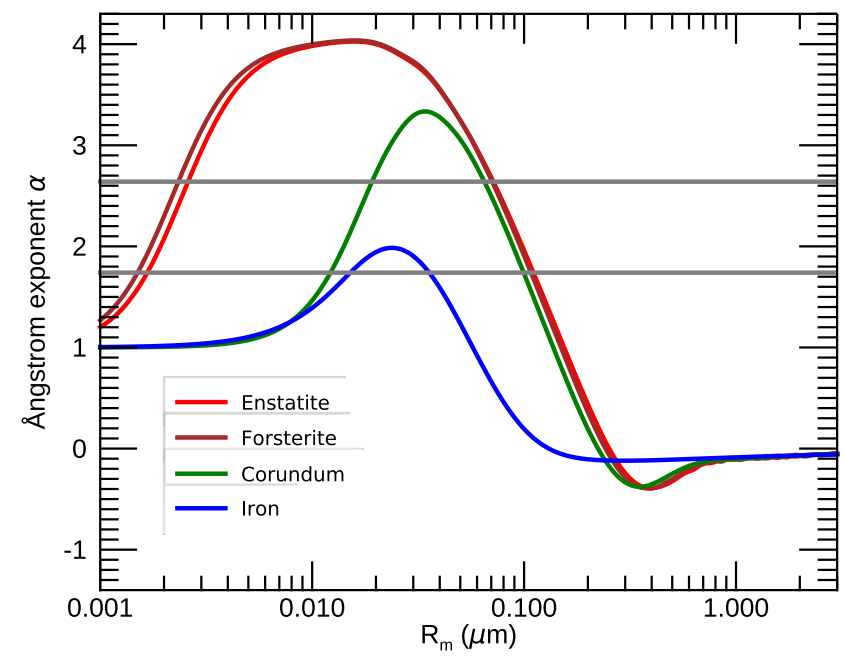

Fig. 4. Calculated absorption Ångström exponents $\alpha$ as a function of particle size for four different minerals. For each mineral we used a lognormal particle size distribution, where $R_{m}$ is the median particle size in the distribution. The two grey horizontal lines denote the $\pm 1 \sigma$ bounds to the Ångström exponent obtained from the GTC observations.

3.1. If we assume that the fitted model is correct, a reduced chi-square of $\approx 1$ should have been obtained, however. In order to achieve this, a second component would have to be added to the quoted flux errors, which is about 1.5 times higher than these. This second component, as well as a variation of $\alpha$ of $\approx 0.15$, observed when different sets of pointings were used for the flux normalisation of Sect. 3, has been included in the quoted error of $\alpha$ (Eq. 4). The quoted error also includes the effects of considering only subsets of the data: using only the even numbered points increases $\alpha$ by 0.5 , whereas the odd points on their own decrease it by 0.4 .

We also note that the reddest band in Fig. 3 has a poorer fit than the other bands. When we omit this band from the analysis, the AAC across the remaining four bands increases to $\alpha=2.30$.

\section{Discussion: absorbers that may cause the dimming events}

The dimming events of KIC8462 that have been observed, with depths of up to $1.6 \%$, have a clear colour signature, with the dips approximately 1.5-2 times deeper in the bluer than in the redder bands. This dependency is analysed in terms of the absorption Angström coefficient (AAC) of the dimmings, found to be $\alpha=2.19 \pm 0.45$, which describes the wavelength dependency of the opacity of an absorbing material, assumed to be optically thin.

In Fig. 4 we show the AACs for several potential absorbing materials. These coefficients are based on Mie extinction cross-sections ${ }^{1}$ for spherical particles with log-normal size distributions around the indicated median sizes $R_{m}$, with the materials' real and imaginary refraction indices given in (Alonso et al. 2016, see also for a plot assuming narrower particle size distributions). The plotted AACs were derived from the cross-sections of the materials at 587 and $830 \mathrm{~nm}$, which are averages between our two bluest and reddest bands, respectively.

\footnotetext{
1 Cross-sections for $\log$ normal distributions were calculated with the set of Mie scattering routines made available by the Earth Observations Data Group of the University of Oxford, at http://eodg.atm.ox.ac.uk/MIE/
}

Figure 4 shows that the range of $\alpha$ from the dimming events is compatible with several combinations of particle sizes and materials. Given the uncertainties in the true nature of the particles' composition, shapes, and size distribtion, we may only conclude that an optically thin absorber must be dominated by particles that are smaller than $\approx 0.15 \mu \mathrm{m}$. In the Raleigh regime, where the particle size is much smaller than the wavelength, the AAC tends towards 1 (Moosmüller 2011), and sizes smaller than $\approx 0.0015 \mu \mathrm{m}$ may also be excluded with reasonable certainty. An optically thin absorber must therefore be dominated by particles with sizes in the Mie regime.

We have to emphasize that the quoted value for the AAC comes from different dimming events, and dust properties may be different in them. It is of note that the measure at the deepest dip (point 13) has a more narrow spread in colour than the second deepest measure (point 8, see Fig. 3). If point 13 is discarded, the AAC increases by 0.38 . Conversely, if point 8 is discarded, the AAC decreases by 0.29 .

The observed AAC has been derived under the assumption of an optically thin absorber. It may well be the case, however, that at least part of the absorbing cross-section is optically thick. In this case, the optically thin part of the cross-section would need to have a higher AAC in order to compensate for the optically thick part of the section, that on its own has a value of $\alpha=0$. The observed value of $\alpha=2.19 \pm 0.45$ is therefore a lower limit. However, as we see in Fig. 4, an increase of $\alpha$ by 1-1.5 does not generate significant new constrains on the absorbing materials, with the exception that iron, owing to its high refractive index, may be excluded.

The observed colour-dependency of the dimming in KIC8462 therefore supports, but does not prove, the hypothesis that occultation by dusty material is the principal origin of the dimming events. Such occulters, in the form of a few distinct compact planetesimals enshrouded in wide dust clouds have been proposed by Neslušan \& Budaj (2017) to be responsible for the dimming events that were observed by Kepler.

Blow-out of dust particles will occur if radiation pressure from the central star becomes a significant fraction of the gravity that determines the particles' orbit. This fraction, known as the $\beta$ parameter (Burns et al. 1979), surpasses a value of $\beta=0.5$ for particles of $\lesssim 1 \mu \mathrm{m}$ (see Fig. 2 of Neslušan \& Budaj 2017). Longer-term brightness variations, if caused by dust particles, may therefore only arise from dust with significantly larger particles, which are inert to blow-out. Following this argumentation, Wyatt et al. (2018) predicted that such variations should be of neutral colour at visible wavelengths (see their Fig. 3).

Meng et al. (2017) indeed reported a gradual fading between October 2015 and December 2016 with a relatively neutral colour. They quoted it in terms of the extinction ratio $R_{V}$ (Cardelli et al. 1989), with $R_{V} \gtrsim 5$. In terms of the AAC, this corresponds $^{2}$ to a value of $\alpha \lesssim 1.1$. For the long-term fading

\footnotetext{
2 We base this conversion on the fact that ratios of absolute extinctions $A_{n} / A_{m}$ are identical to the depth ratios used in our work; that is, $A_{n} / A_{m}=D_{n} / D_{m}$ as long as the $A$ and $D$ are $<<1$, with $n$ and $m$ representing two wavelengths. Cardelli et al. (1989) consolidated the use of $A(\lambda) / A_{V}$ as a function of $R_{V}$ to describe colour dependence of interstellar extinction through a single number $R_{V}$. For a given $R_{V}$ and a set of wavelengths $\lambda_{i}$, we may then calculate all values $A\left(\lambda_{i}\right) / A_{V}$ using their Eq. (3). Through $\chi^{2}$ minimisation, we may then find the value $\alpha$ that generates the set of $D\left(\lambda_{i}\right) / D_{V}=\left(\lambda_{i} / \lambda_{V}\right)^{-\alpha}$ that most closely matches the set of the $A\left(\lambda_{i}\right) / A_{V}$. This procedure can easily be reversed to determine $R_{V}$ for a given $\alpha$. We note that both $R_{V}$ and the AAC generate phenomenological descriptions of wavelength dependencies without implying underlying physical models.
} 
during the Kepler mission (Montet \& Simon 2016), a similar value of $R_{V}=5.0 \pm 0.9$ or $\alpha=1.1 \pm 0.1$ was also quoted by Davenport et al. (2018), based on near-UV photometry acquired in 2011 and 2012 by the GALEX mission, which they compared to Kepler photometry. The long-term fading may therefore have a much weaker colour dependence than the short events observed by us and by B18. However, the colours reported by Meng et al. (2017) were derived from only two epochs, comparing fluxes before and after an observational gap in winter 2015/16, while the colours reported by Davenport et al. (2018) were based on a wavelength interval rather different from the one used in our work. We consider it therefore too early to ascertain that the longer-term flux variations have a fundamentally different origin (or very different absorbers) than the short dimming events. Continued longer-term coverage of KIC8462 in multiple wavelengths is certainly desirable to clarify whether a difference exists between the colours of the dimming events lasting a few days and the variations on timescales of months and longer.

Considering again the short dimming events, Wyatt et al. (2018) inferred from the duration of the deepest events in the Kepler data that any occulters causing them have to be on highly eccentric orbits with pericenters at $<0.6$ AU. This would imply still larger sizes for dust particles, in order to become resistent to blow-out. Our data, and those used by B18 as well, stipulate that the dimmings are generated by smaller dust particles, however, with $R_{m} \lesssim 0.15 \mu$ (this work) or $R_{m} \lesssim 0.2 \mu$ (B18).

For fine dust like this, radiation pressure is higher than gravitational attraction by the $\operatorname{star}(\beta>1)$. Dust with only weak gravitational bounding to an orbiting body will therefore decouple from it and move on a hyperbolic trajectory away from the star and the orbiter (see Fig. 3 of Neslušan \& Budaj 2017). This decoupling is rather fast: at a distance of 0.6AU from KIC8462, acceleration from radiation pressure will move dust with $\beta=1$ within about 3.5 days over a distance of the KIC8462 radius (1.58 $\left.R_{\odot}, \mathrm{B} 18\right)$. When we assume a dust size of $0.1 \mu \mathrm{m}, \beta$ may take values of $2-10$, depending on the material, and the time to cover the same distance will be reduced by a factor of $\beta^{1 / 2}$. The presence of such fine dust during the short dimming events of KIC8462 therefore requires that this dust is constantly replenished during the orbit of the occulter. We can therefore exclude an occulter that only consists of dust, and instead require a central object that is able to regenerate this dust during a flyby on KIC8462.

If dust is indeed the source of dips in KIC8462, it is also to be expected that the observation of much deeper dips like those found in the Kepler data will show that these have a weaker colour signature. The reason is that a dimming depth of 15-20\% is difficult to generate by an absorber that is optically thin across the entire occulted stellar surface. In order to be optically thin, such a strong absorber would have to be of rather uniform optical thickness, with values of the order of $\tau \approx 0.1$ on a size scale of the stellar diameter. More likely, such an absorber has significant non-uniformities, and at least part of it will be optically thick during the deeper events.

The observational coverage of a deep event with simultaneous multicolour photometry or with spectrophotometry, and preferentially with a dense temporal coverage, should therefore be central to resolve the hypothesis of a mostly dusty absorber as the source of the dimming events in KIC 8462852.

\section{Summary}

The principal results of this work are summarised below.

- In dimming events of KIC8462 of depths of up to $1.6 \%$ and lasting a few days, we find a clear colour signature, with dips approximately 1.5-2 times deeper in the bluer than in the redder visual bands. The depths' wavelength dependency can be described by a single number, an absorption Ångström coefficient of $2.19 \pm 0.45$.

- This is different to the flux variations of KIC8462 on timescales of months to years, which have been reported to display largely neutral colours in the visual regime. These variations are probably caused by different absorbers, or arise from processes that are different from those causing the short events.

- Assuming that the short events are caused by occultations, their wavelength dependency implies that most of the crosssection of the occulters must be optically thin and that they consist of particles with sizes in the range $0.0015-0.15 \mu \mathrm{m}$.

- Small particles like this will not resist blow-out by radiation pressure. At expected periastron distances of $0.6 \mathrm{AU}$ or less, radiation pressure will cause them to deviate on timescales of days over distances larger than the radius of the central star. They must therefore be replenished continually.

- In the 15-20\% deep events observed to date by Kepler alone, a significant part of their occulting cross-section was likely optically thick. Hence we predict a tendency towards more neutral colour signatures for deeper events. This tendency may already be present in the deepest event that was observed by the GTC.

Acknowledgements. We thank the referee, Benjamin T. Montet of the California Institute of Technology, for the rapid and thorough revision, which led to a marked improvement in the presentation of this work. This research made use of data acquired with the Gran Telescopio Canarias (GTC), installed at the Spanish Observatorio del Roque de los Muchachos of the Instituto de Astrofísica de Canarias, on the island of La Palma. We also thank the staff of GTC for its excellent handling of our observing requests. HD, RA, and DNe acknowledge support by grants ESP2015-65712-C5-4-R, ESP2016-80435C2-2-R, and RYC-2010-06519 of the Spanish Secretary of State for R\&D\&i (MINECO).

\section{References}

Alonso, R., Rappaport, S., Deeg, H. J., \& Palle, E. 2016, A\&A, 589, L6 Ångström, A. 1929, Geogr. Ann., 11, 156

Bodman, E. H. L., \& Quillen, A. 2016, ApJ, 819, L34

Boyajian, T. S., LaCourse, D. M., Rappaport, S. A., et al. 2016, MNRAS, 457, 3988

Boyajian, T. S., Alonso, R., Ammerman, A., et al. 2018, ApJ, 853, L8

Brown, T. M., Latham, D. W., Everett, M. E., \& Esquerdo, G. A. 2011, AJ, 142, 112

Burns, J. A., Lamy, P. L., \& Soter, S. 1979, Icarus, 40, 1

Cardelli, J. A., Clayton, G. C., \& Mathis, J. S. 1989, ApJ, 345, 245

Davenport, J. R. A., Covey, K. R., Clarke, R. W., et al. 2018, ApJ, 853, 130

Meng, H. Y. A., Rieke, G., Dubois, F., et al. 2017, ApJ, 847, 131

Montet, B. T., \& Simon, J. D. 2016, ApJ, 830, L39

Moosmüller H. 2011, Atm. Chem. Phys., 11, 10677

Neslušan, L., \& Budaj, J. 2017, A\&A, 600, A86

Schaefer B. E. 2016, ApJ, 822, L34

Wright, J. T., \& Sigurdsson, S. 2016, ApJ, 829, L3

Wyatt, M. C., van Lieshout, R., Kennedy, G. M., Boyajian, T. S. 2018, MNRAS, 473,5286 